\title{
Basics of EHL for practical application
}

\author{
Hugh Spikes \\ Tribology Group, Department of Mechanical Engineering, Imperial College London, UK \\ h.spikes@imperial.ac.uk
}

\section{SUMMARY}

Elastohydrodynamic lubrication (EHL) is present in all lubricated components whose elements roll together, including gears, rolling bearing, cams and constant velocity joints. These are characterised by having very localised and thus very high pressure contact, of order 1 to $3 \mathrm{GPa}$, between the elements.

Two important practical properties of EHL contacts are the lubricant film thickness and the friction, and lubricant and machine designers and users need to be able to predict both of these. In principle they can be determined from full numerical solution of the elastohydrodynamic problem. However this is quite difficult and time-consuming and requires detailed knowledge of the rheology of the lubricant film at high pressure and shear rate. This paper is aimed at practising engineers and describes alternative approaches, i.e. how EHL can be applied to predict film thickness and friction in practical applications.

\section{INTRODUCTION}

Lubricated machine components can be divided into those whose elements slide together and those having elements that roll against one another. In the former, such as piston ring/liners and plain bearings, the elements are generally designed to conform closely, so as to spread the applied load over a large fluid-film area and thus reduce the contact pressure. By contrast, in rolling-sliding components such as rolling bearings, involute gears, cam/follower systems and constant velocity joints, as shown in Fig. 1, the elements must have curved surfaces and be non-conforming so as to be able to roll. If these contacting elements are metallic or ceramic and so have a high elastic modulus, the resulting contacting area is very small. In consequence these contacts experience very high pressures, often of the order of several gigapascals $(1 \mathrm{GPa}=10 \mathrm{kbar})$.

The lubrication of conforming lubricated contacts is well described by the hydrodynamic lubrication theory first developed by Reynolds in 1886 [1]. However, for many years after this it was not understood how non-conforming contacts could operate successfully, since Reynolds' hydrodynamic theory taken alone predicts only very thin lubricating films in such high pressure contacts. Then, in the early 1940s, Ertel showed that two beneficial effects of the very high contact pressure, the local elastic deformation of the contacting surfaces and an increase in lubricant viscosity with pressure in the contact, couple with hydrodynamic theory to promote lubricant film formation in high pressure contacts [2].

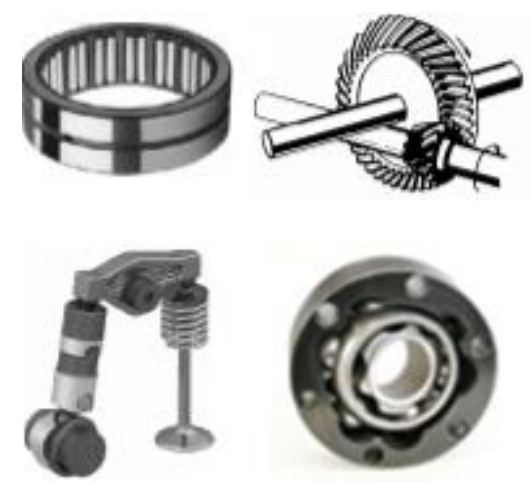

Fig. 1. Types of EHD contact

This type of hydrodynamic lubrication is now known as elastohydrodynamic lubrication (EHL) and the lubricating films formed in such contacts are called elastohydrodynamic (EHD) films. In the paper the abbreviations EHL and EHD will be used interchangeably. A history of the development of our current understanding of EHL is given in [3].

It is important to appreciate the main features of EHL and to be able to predict the key properties of EHD films; their film thickness, friction and pressure. Of particular concern nowadays is to be able to predict and thus, via machine component and lubricant design, to minimise EHD friction. This paper will outline some of the main features of EHL 
and especially how to determine EHD film thickness and friction. The application of EHL to the analysis of bearing and gear contacts will then be briefly outlined. The aim is not provide a design guide but just to show how application of EHL can be used to assess the influence of lubricants on film thickness and friction in such components. It should be noted that this paper is confined to full-film EHD conditions, where the lubricant film thickness is several times greater than the surface roughness so that roughness has negligible impact on EHD film formation or on friction.

\section{THEORETICAL BACKGROUND}

Mathematical solution of the isothermal elastohydrodynamic lubrication problem to determine lubricant pressure and film thickness involves simultaneous solution of five equations; 1, Reynolds' hydrodynamic equation; 2, geometry of the surface to describe how fluid film thickness, $h$ varies with $x, y$ across the contact; 3 , an equation balancing the applied load on the contact, $W$, with the fluid film pressure, $p ; 4$, elastic deformation, $\delta$, of the contacting surfaces due to pressure; 5 , dependence of lubricant viscosity, $\eta$, on pressure. These equations are shown respectively for a steadilyloaded, elliptical contact below [4].

$$
\begin{aligned}
& \frac{\partial}{\partial x}\left\{\frac{h^{3}}{\eta} \frac{\partial p}{\partial x}\right\}+\frac{\partial}{\partial y}\left\{\frac{h^{3}}{\eta} \frac{\partial p}{\partial y}\right\}=12 U \frac{d h}{d x} \\
& h=h_{c}+\frac{x^{2}}{2 R_{x}}+\frac{y^{2}}{2 R_{y}}+\delta(x, y) \\
& W=\iint_{A} p d x d y \\
& \delta(x, y)=\frac{1}{2 \pi E^{\prime}} \iint_{A} \frac{p\left(x^{\prime}, y^{\prime}\right)}{r} d x^{\prime} d y^{\prime} \\
& \eta=f(p)
\end{aligned}
$$

In these equations, $U, R_{x}, R_{y}$ and $E$ ' are combined values of the speeds of the two surfaces, their radii and their elastic moduli respectively. The way these are combined is described later in this paper. $h_{c}$ is the lubricant film thickness at the centre of the contact. For accurate solution, an equation describing the dependence of lubricant density on pressure should also be included, but this generally has much a smaller impact on the solution than other factors.

Simultaneous solution of equations (1) to (5) yields the film thickness and pressure of the lubricant film across the contact. A separate pair of equations is then used to calculate the friction, $F$ of the film;

$$
\begin{aligned}
& F=\frac{1}{2} \int_{A} h \frac{d p}{d x} d A+\int_{A} \tau d A \\
& \tau=f(\eta, \dot{\gamma})
\end{aligned}
$$

where the two terms in equation (6) represent rolling friction and sliding friction respectively. $\eta$ is the low shear rate viscosity (which depends on pressure), $\tau$ is the shear stress of the fluid film under Couette shear and $\dot{\gamma}$ is the strain rate (the sliding speed between the two surfaces divided by the local film thickness).

We can explore the impact of elastic deformation and lubricant viscosity-pressure response on EHL film thickness by using various combinations of the above equations. Consider the contact of a $10 \mathrm{~mm}$ steel ball rolling with speed $2 \mathrm{~m} / \mathrm{s}$ against the flat surfaces of a rotating steel disc, with applied load $100 \mathrm{~N}$, lubricated by an oil of viscosity $200 \mathrm{cP}(0.2$ Pas) and pressure-viscosity coefficient $10 \mathrm{GPa}^{-1}$. Both ball and disc have elastic modulus of $210 \mathrm{GPa}$ and Poisson's ratio 0.3 , typical of bearing steels.

Table 1 compares the minimum film thickness formed in the four cases of; (i) classical hydrodynamic lubrication (pressure-viscosity and elastic deformation effects negligible); (ii) piezoviscous effects significant but elastic deformation negligible; (iii) elastic deformation significant but piezoviscous effects negligible and (iv) EHL, with both piezoviscous effects and elastic deformation playing a role. 


\begin{tabular}{|c|c|c|c|}
\hline $\begin{array}{c}\text { Hydrodynamic } \\
\text { lubrication }\end{array}$ & - & Soft EHL & EHL \\
\hline $\begin{array}{c}\text { Isoviscous- } \\
\text { rigid } \\
\delta(\mathrm{x}, \mathrm{y})=0\end{array}$ & $\begin{array}{c}\text { Piezo- } \\
\text { viscous- } \\
\text { rigid, } \\
\delta(\mathrm{x}, \mathrm{y})=0\end{array}$ & $\begin{array}{c}\text { Iso-viscous- } \\
\text { elastic }\end{array}$ & $\begin{array}{c}\text { Piezo-viscous- } \\
\text { elastic }\end{array}$ \\
\hline Eqs. (1)(2)(3) & $(1)(2)(3)(5)$ & $(1)(2)(3)(4)$ & $(1)(2)(3)(4)(5)$ \\
\hline $0.3 \mathrm{~nm}$ & $10 \mathrm{~nm}$ & $150 \mathrm{~nm}$ & $570 \mathrm{~nm}$ \\
\hline
\end{tabular}

Table 1. Minimum EHD film thickness in four regimes of hydrodynamic lubrication

The beneficial effect on EHL film thickness of having both elastic deformation and enhancement of lubricant viscosity with pressure is evident

\section{EHD CONTACT PRESSURE AND CONTACT DIMENSIONS}

Accurate determination of EHD fluid film pressure and contact size requires full numerical solution of the pressure, viscosity and deformation equations. However for many purposes, an acceptable approximation is to assume that the contact has the pressure distribution and size given by the Hertz equations [5]. These predict a parabolic pressure distribution for a line contact and an ellipsoidal one for a point contact as shown in Fig 2.

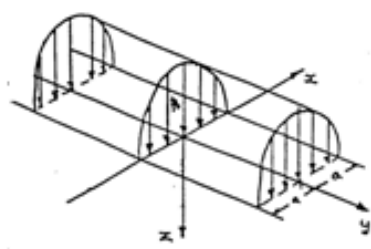

Line contact

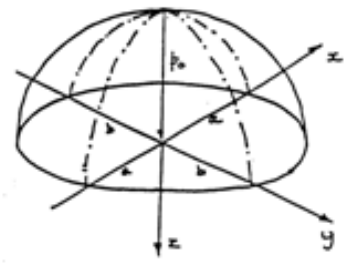

Point contact

Fig. 2. Hertz pressure distribution in line and point contacts

The maximum Hertz pressure, and also the contact dimensions for a line contact and circular point contact can be calculate from the equations in Table 2

\begin{tabular}{|l|c|c|}
\hline & Line Contact & $\begin{array}{c}\text { Circular Point } \\
\text { Contact }\end{array}$ \\
\hline $\begin{array}{l}\text { Half-contact } \\
\text { width or } \\
\text { contact } \\
\text { radius }\end{array}$ & $a=2\left(\frac{\left.2 W_{L^{\prime} R}\right)^{1 / 2}}{\pi E^{\prime}}\right)$ & $a=\left(\frac{3}{2} \frac{W R}{E^{\prime}}\right)^{1 / 3}$ \\
\hline $\begin{array}{l}\text { Maximum } \\
\text { contact } \\
\text { pressure }\end{array}$ & $p_{0}=\left(\frac{W_{L} E^{\prime}}{2 \pi R}\right)^{1 / 2}$ & $p_{0}=\left(\frac{1}{\pi}\right)\left(\frac{3 W E^{\prime 2}}{2 R^{2}}\right)^{1 / 3}$ \\
\hline
\end{tabular}

Table 2. Contact pressure and dimensions from Hertz theory

In this table, $W_{L}$ is the applied load per unit length (line contact), $W$ is total applied load (point contact), $p_{o}$ is the maximum Hertz pressure and $a$ is the predicted half-width (line contact) or contact radius (point contact). $E^{\prime}$ and $R$ are the reduced elastic modulus and the reduced radius respectively. These combine the individual elastic moduli and radii of the two surfaces $(1,2)$ according to equations 8 and 9 respectively, where $E$ is the elastic modulus and $v$ is the Poisson's ratio of the surfaces;

$$
\begin{aligned}
& \frac{2}{E^{\prime}}=\left(\frac{1-v_{1}^{2}}{E_{1}}+\frac{1-v_{2}^{2}}{E_{2}}\right) \\
& \frac{1}{R}=\frac{1}{R_{1}}+\frac{1}{R_{2}}
\end{aligned}
$$

For a concave surface $R_{1}$ or $R_{2}$ is negative while for a flat surface $R_{1}$ or $R_{2}$ is infinite. 
It should be noted that many textbooks use a different version of combined elastic modulus, often denoted $E^{*}$, which has a value half that of $E$ '.

The equations given in Table 2 apply only to the two special cases of line or circular point contact. If the two surfaces are curved by different amounts in orthogonal directions (e.g. a spherical roller bearing or a ball bearing in a raceway groove), then we must calculate the reduced radii in orthogonal directions;

$$
\begin{aligned}
& \frac{1}{R_{x}}=\frac{1}{R_{1 x}}+\frac{1}{R_{2 x}} \\
& \frac{1}{R_{y}}=\frac{1}{R_{1 y}}+\frac{1}{R_{2 y}}
\end{aligned}
$$

where $x$ is conventionally the rolling direction. If $R_{x}$ and $R_{y}$ are unequal but finite, then the contact patch is elliptical and we set $R$ to the geometric mean radius:

$$
R=\left(R_{x} R_{y}\right)^{1 / 2} \text {. }
$$

\begin{tabular}{|l|l|}
\hline \multicolumn{2}{|l|}{$\begin{array}{l}\text { Elliptical Contact } \\
\left.\text { (dimensions } 2 a \times 2 b ; R=\left(R_{x} R_{y}\right)^{1 / 2}\right)\end{array}$} \\
\hline $\begin{array}{l}\text { "Effective contact } \\
\text { half-width" } \\
c \equiv \sqrt{a b}\end{array}$ & $c=\left(\frac{3}{2} \frac{W R}{E^{\prime}}\right)^{1 / 3} F_{1}$ \\
\hline $\begin{array}{l}\text { Maximum contact } \\
\text { pressure }\end{array}$ & $p_{0}=\left(\frac{1}{\pi}\right)\left(\frac{3 W E^{\prime 2}}{2 R^{2}}\right)^{1 / 3} F_{1}^{-2}$ \\
\hline
\end{tabular}

Table 3. Hertz contact dimensions and pressure for elliptical contact

If the ratio of the radii $R_{x} / R_{y}$ is less than about 5 (and greater than 1/5) we may obtain an approximate solution by putting $F_{l}=1$ and the ratio of contact half-widths to $b / a=\left(R_{x} / R_{y}\right)^{-2 / 3}$. Combination of this last equation with that for the effective contact half-width in Table 3 enables both $a$ and $b$ to be calculated. If $R_{x} / R_{y}$ is greater than 5 then graphs relating $F_{l}$ and $b / a$ to $R_{x} / R_{y}$ are available [5].

Use of the above tables enables the maximum contact pressure, $p_{o}$, and the contact dimensions to be calculated. In EHL contacts, $p_{o}$ is usually in the range 0.5 to $4 \mathrm{GPa}$ while $2 a$ is typically 100 to $600 \mu \mathrm{m}$. The mean pressure can, of course, be calculated from the applied load divided by the contact area but is also given by $\pi / 4 p_{o}$ for line and $2 / 3 p_{o}$ for circular contact.

For EHD contacts, the above equations are only approximate, especially for pressure. Numerical solution of the EHD problem yields pressure distributions of the form shown in Fig. 3, as compared to the parabola predicted by Hertz for a static, dry contact and shown in Fig. 2. There is a build-up of pressure in the contact inlet upstream of the central flat region (which is crucial in generating a fluid film) and a pressure maximum (known as a pressure spike), followed by a very rapid collapse of pressure at the contact exit.

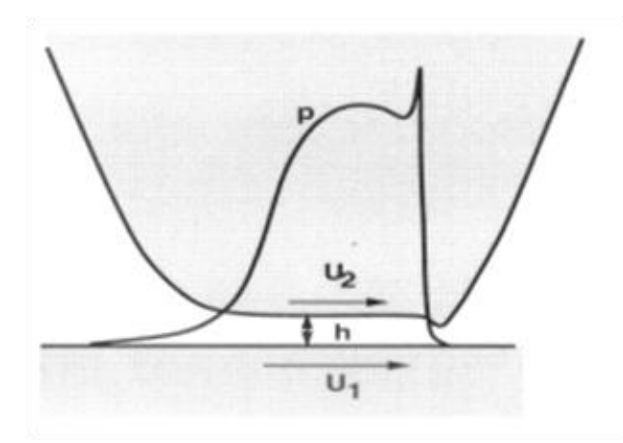

Fig. 3. Typical EHD pressure profile 


\section{EHD SPEEDS}

A key feature of almost all practical EHD contacts (as opposed to many bench tests such as the 4-ball and block on ring test) is that the contacting surfaces roll together. In many cases they also slide and so experience mixed sliding-rolling. In analysing an EHD contact there are thus two important and quite different speeds. One is the mean of the rolling speeds of two surfaces with respect to the contact;

$$
U=\frac{u_{1}+u_{2}}{2}
$$

This is also called the entrainment speed and is the speed with which lubricant is drawn or "entrained" into the contact. It thus determines the EHD film thickness and is the speed appearing in equation 1.

The second is the sliding speed, which is the speed of the two surfaces relative to each other;

$$
u_{s}=\left|u_{1}-u_{2}\right|
$$

This speed controls the friction and also the heat generation but has very little impact on the film thickness. In EHL, the slide-roll-ratio $(S R R)$, which is the ratio of the two speeds, is commonly employed;

$$
\operatorname{SRR}=\frac{\left|u_{1}-u_{2}\right|}{\left(u_{1}+u_{2}\right) / 2}
$$

This has value 0 in "pure rolling" when $u_{1}=u_{2}$, and value 2 in "pure sliding" when $u_{2}=0$. If the two surfaces move in opposite directions with respect to the contact then it is possible for SRR to be much greater than 2, with very low entrainment speeds but high sliding speeds. This occurs in some cam-follower contacts.

\section{DECOUPLING OF EHD FILM THICKNESS AND FRICTION}

Typical film shapes are shown for a circular point EHD contact (ball on flat) and a line EHD contact (roller on flat) in the experimental optical interference images in Fig. 4. In both images the inlet is on the left. There is quite constant film thickness across most of the contact with a reduced film thickness or "constriction" at the rear and sides. This is also shown in Fig. 3. The film thickness at this constriction is $c a 70 \%$ of the central film thickness and tends to be thinner at the sides than at the rear of the contact. The lubricant film cavitates as it emerges from the rear of the contact, as can be seen on the right hand side of both images

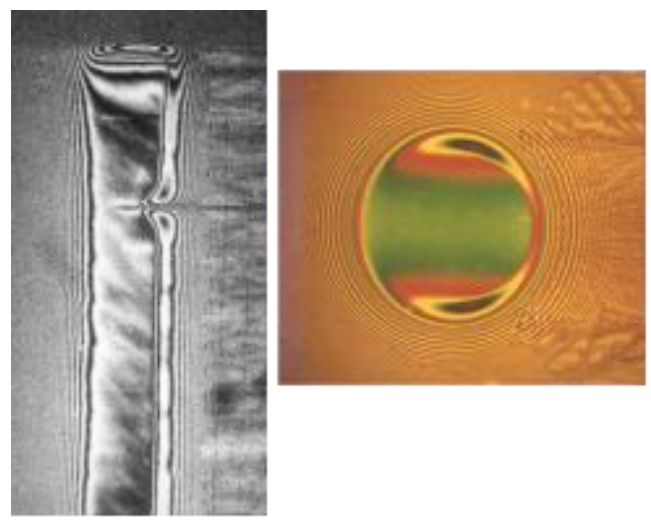

Fig. 4. Optical interference images of line and point EHD contacts [3]

One of the most important features of EHL is that, unlike conventional hydrodynamic lubrication, there is no direct relationship between the EHD film thickness and the EHD friction. The reason for this comes from the shape of the EHD film. The quantity of lubricant entrained is determined primarily by the geometry and properties of the lubricant in the critical inlet region that spans a zone from about distance $a$ (the contact half-width or radius) in front of the inlet edge of the contact flat to this inlet edge itself. In this region the pressure rises slowly from atmospheric to about 150 $\mathrm{MPa}$ and the quantity of lubricant entrained, and thus the film thickness, depends on the viscosity of the lubricant over this pressure range. The prevailing strain rate in the inlet is determined by recirculation of lubricant and is usually $c a$ $10^{6} \mathrm{~s}^{-1}$. Under these relatively low pressure conditions most base fluids are Newtonian, so film thickness depends on the Newtonian but piezo-viscous properties of the lubricant. The EHD film is so thin that once within the contact the lubricant has no option but to progress through to the exit - there is negligible side-flow. This means that the film thickness does not depend on the properties of the lubricant film within the flat, central region of the contact. 
By contrast, the friction depends almost entirely on the properties of the lubricant film within the flat central contact region since this part of the film is highly pressurised and thus has high shear stress. In this central region, so long as some sliding is present, the lubricant experiences both very high pressures and high strain rates and its response is always highly non-Newtonian, even for simple liquids. In practice the lubricant shear thins towards a value that depends strongly on the shape and flexibility of the lubricant molecules and only slightly on the lubricant's original viscosity.

This means that, unlike in conventional hydrodynamic lubrication, we can design for EHD film thickness and EHD friction independently, for example obtaining a combination of high film thickness and low friction, by appropriate lubricant selection.

\section{EHD FILM THICKNESS}

\subsection{EHD Film Thickness Calculation}

There are no useful analytical solutions of the EHL problem and instead we tend to use regression-fitted equations that have been developed from repeated numerical solution of the EHL problem over a wide range of contact and material conditions. For generality (and to limit the number of solutions required to generate the regression equations) most of these solutions employ three non-dimensional groups;

speed parameter, $\bar{U}=\frac{U \eta_{o}}{E^{\prime} R_{x}}$

materials parameter, $\bar{G}=\alpha E^{\prime}$

load parameter, $\bar{W}=\frac{W}{E^{\prime} R_{x}^{2}}$ (elliptical/point contact), $\overline{W_{L}}=\frac{W}{E^{\prime} R_{x} L}$ (line contact)

where $\eta_{o}$ is the dynamic viscosity of the lubricant at atmospheric pressure while $\alpha$ is a composite pressure-viscosity coefficient value over the contact inlet, the " $\alpha$-value". $R_{x}$ is the reduced radius in the entrainment direction and is calculated from Equ. 10

Many alternative EHL film thickness equations have been developed. Two that apply to line contact are due to Dowson and Higginson [6] and Dowson and Toyoda [7] for minimum and central film thickness respectively;

$$
\begin{aligned}
& \frac{h_{o}}{R_{x}}=2.65 \bar{U}^{0.7} \bar{G}^{0.54} \bar{W}_{L}^{-0.13} \\
& \frac{h_{c}}{R_{x}}=3.11 \bar{U}^{0.69} \bar{G}^{0.56} \bar{W}_{L}^{-0.1}
\end{aligned}
$$

A set of four equations for point contact due to Chittenden et al. [8] is given below. These apply to all elliptical contacts, with separate equations for cases where entrainment is along the major and the minor axis respectively. Equations for entrainment at any angle are given in [9]. Equations are given for both central film thickness, $h_{c}$ and minimum film thickness, $h_{o}$.

$R_{x} \geq R_{y}$ (Long ellipse axis is along rolling/ entrainment direction)

$$
\begin{aligned}
& \frac{h_{o}}{R_{x}}=3.00 \bar{H} *\left(1-e^{-0.96\left(R_{y} / R_{x}\right)}\right) \\
& \frac{h_{c}}{R_{x}}=3.06 \bar{H} *\left(1-e^{-3.36\left(R_{y} / R_{x}\right)}\right)
\end{aligned}
$$

$R_{x} \leq R_{y}$ (Long ellipse axis is transverse to rolling/ entrainment direction)

$$
\begin{aligned}
& \frac{h_{o}}{R_{x}}=3.63 \bar{H} *\left(1-e^{-0.67\left(R_{y} / R_{x}\right)}\right) \\
& \frac{h_{c}}{R_{x}}=4.30 \bar{H} *\left(1-e^{-1.30\left(R_{y} / R_{x}\right)}\right)
\end{aligned}
$$

where;

$\bar{H}^{*}=\bar{U}^{0.68} \bar{G}^{0.49} \bar{W}^{-0.073}$ 
It is instructive to reduce these equations to dimensional form and for Equ. (22) this gives;

$$
h_{o}=3.63 \frac{\left(U \eta_{o}\right)^{0.68} \alpha^{0.49} R_{x}^{0.47}}{E^{\prime^{0.12}} W^{0.073}}\left(1-e^{-0.67\left(R_{y} / R_{x}\right)^{2 / 3}}\right)
$$

Thus the film thickness increases quite strongly with entrainment speed and viscosity and less strongly with radius and pressure viscosity coefficient. It is only weakly dependent on applied load. This is because variation of load and elastic modulus change the overall contact area but have little effect on the shape of the inlet. It is almost independent of sliding speed to to SRR values of 50\%. Although three non-dimensional parameters, $\bar{U}, \bar{G}$ and $\bar{W}$, are most widely use in EHD film thickness analysis, only two are actually required and some research groups prefer equations based on just two [10].

It is important to note that the above film thickness equations are only applicable to high pressure lubricated contacts where elastic deformation and lubricant piezoviscous response are both significant so the contact operates in the EHD regime. For non-conforming, compliant solids such as polymers, or with lubricants having very low pressure viscosity coefficients such as water, elastic deformation occurs without there being any significant rise in viscosity in the inlet. The contact then operates in the isoviscous-elastic hydrodynamic regime and a different set of film thickness equations should be used. Low pressure contacts having negligible elastic deformation or piezoviscous response operate in the conventional hydrodynamic regime, as is the case with plain bearings, piston/liner contacts and some high speed, lightly-loaded gears. Charts are available for both line [11] and elliptical [12] contact from which to determine the prevailing hydrodynamic lubrication regime.

\subsection{EHD Film Thickness Measurement}

Historically, many methods have been developed for measuring EHD film thickness, but nowadays the main ones are capacitance, ultrasonics, optical interference and fluorescence. The first two can be used in real machine elements and tend to be employed to test design or lubricant supply issues. The last two require one surface to be opticallytransparent (usually glass or sapphire) and are thus not practicable to measure film thickness between a pair of real machine elements. However they are well-suited to study the EHL film-forming properties of lubricants. Most methods have been discussed in [13] while a description of the ultrasonic method, which is relatively recent, can be found in [14].

The optical interference method, which was used to generate the images in Fig. 4, has become a key tool for studying the film-forming properties of lubricants and greases. Fig. 5 shows a plot of measured film thickness versus speed for an ester oil measured at three test temperatures.

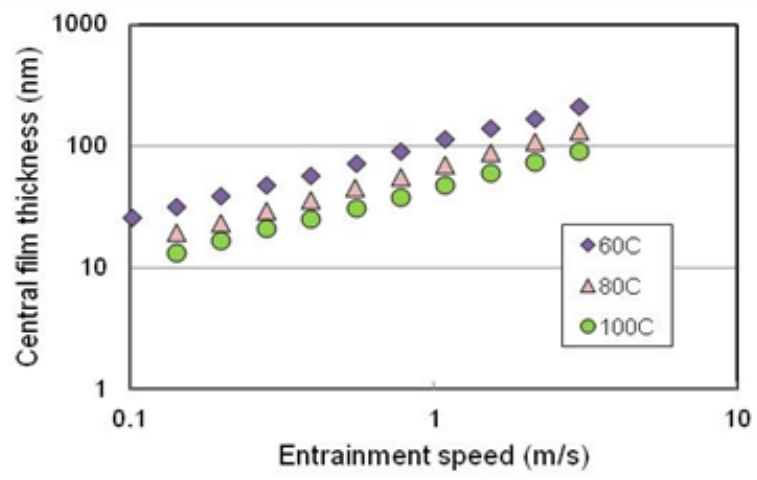

Fig. 5. Typical $\log ($ film thickness) versus $\log ($ entrainment speed) results, (lubricant is di-2-ethylhexylphthalate)

EHD film thickness equations such as (20)-(23) predict that film thickness $h_{c}$ should be proportional to $\left(U \eta_{o}\right)^{0.68}$, so that at a fixed temperature, $\log \left(h_{c}\right)$ versus $\log (U)$ should give a straight line of gradient 0.68 , as is indeed seen in Fig. 5 . In practice, for polymer-free lubricants this gradient usually lies between 0.6 and 0.75 . As temperature is increased film thickness is reduced, since both viscosity and pressure-viscosity coefficient decrease with increasing temperature.

\subsection{Pressure Viscosity Coefficients}

As indicated in equations (18)-(23) two lubricant properties are required for EHD film thickness caculation, the dynamic viscosity and the composite pressure-viscosity coefficient, both at the prevailing inlet temperature which, in most cases, can be taken as the bulk lubricant temperature. Dynamic viscosity is readily available but pressure viscosity coefficient values are harder to come by. 
Pressure viscosity coefficient, $\alpha$ is defined formally as the fractional rate of change in viscosity with pressure, i.e. $\frac{1}{\eta} \frac{d \eta}{d p}$

. This often varies with pressure and for EHD film thickness calculations we need a composite value over the varying pressure of the contact inlet, the $\alpha$-value. At the relatively low pressures present in EHD contacts inlets (up to 150 $\mathrm{MPa}), \alpha$ is often constant with pressure, so simple integration yields the Barus equation

$\eta(p)=\eta_{o} e^{\alpha p}$

If viscosity-pressure data is available and $\log (\eta)$ versus $p$ is linear, the $\alpha$-value for use in equations (18)-(23) is then simply the slope of $\log (\eta)$ versus $p$. If this slope is not constant then a composite $\alpha$-value devised by Blok, formally termed the reciprocal of the asymptotic isoviscous pressure and having symbol $\alpha^{*}$, is often used [15];

$$
\alpha^{*}=1 / \int_{0}^{\infty} \frac{\eta_{0}}{\eta(p)} d p
$$

In practice, it is normally much easier to estimate the required"effective" $\alpha$-value of a lubricant from optical EHD film thickness measurements, such as those in Fig. 5, than to measure it using high pressure viscometry and equation (27). It can be determined directly from Equ. (22) so long as all the other variables are known. However, more commonly the EHD film thickness formed by a test lubricant is compared to that formed by a reference lubricant of known effective $\alpha$-value under identical test conditions. According to the EHD film thickness equations, at a fixed load and geometry the central film thickness should depend on viscosity and $\alpha$-value according to;

$h_{c} \propto\left(U \eta_{o}\right)^{0.68} \alpha^{0.49}$

So, for a test and reference oil whose film thicknesses are measured at the same entrainment speed;

$$
\frac{h_{\text {test }}}{h_{\text {ref }}} \propto\left(\frac{\eta_{\text {otest }}}{\eta_{\text {oref }}}\right)^{0.68}\left(\frac{\alpha_{\text {test }}}{\alpha_{\text {ref }}}\right)^{0.49}
$$

Table 4 lists some effective $\alpha$-values calculated from EHD film thickness measurements using Equ. (29) for a range of base oils at two temperatures.

\begin{tabular}{|c|c|c|c|c|}
\hline & $\begin{array}{c}\text { Viscosity } \\
\mathrm{cP} \\
60^{\circ} \mathrm{C}\end{array}$ & $\begin{array}{c}\text { Viscosity } \\
\mathrm{cP} \\
100^{\circ} \mathrm{C}\end{array}$ & $\begin{array}{c}\text { Effective } \\
\alpha \text {-value } \\
\mathrm{GPa}^{-1} \\
60^{\circ} \mathrm{C}\end{array}$ & $\begin{array}{c}\text { Effective } \\
\alpha \text {-value } \\
\mathrm{GPa}^{-1} \\
100^{\circ} \mathrm{C}\end{array}$ \\
\hline Di-ethylhexyl-phthalate & 11.59 & 3.89 & 17 & 14 \\
\hline Bis(2-ethyl hexyl)sebacate & 6.00 & 2.73 & 9 & 7 \\
\hline Di-isodecyl azelate & 8.96 & 3.81 & 14 & 13 \\
\hline NPG di-oleate & 13.33 & 5.54 & 13 & 13 \\
\hline NPG di-isostearate & 19.59 & 6.88 & 15 & 14 \\
\hline PAO & 11.52 & 4.17 & 17 & 15 \\
\hline PAO & 16.78 & 5.57 & 17 & 15 \\
\hline PAO & 32.67 & 9.50 & 18 & 15 \\
\hline Group I MO & 13.17 & 4.59 & 24 & 17 \\
\hline Group II MO & 12.81 & 4.48 & 20 & 15 \\
\hline Group III MO & 12.10 & 4.93 & 8 & 9 \\
\hline Group III MO & 18.22 & 6.12 & 25 & 21 \\
\hline Castor oil & 89.37 & 20.19 & 9 & 8 \\
\hline
\end{tabular}

Table 4. Effective pressure-viscosity coefficients measured using optical interferometry (N.B. method is only accurate to $\pm 10 \%$ )

There is a spread of values, but for most fluids $\alpha$-value tends to lie in the range 7 to $25 \mathrm{GPa}-1$. It decreases with temperature and, within a given base stock class, generally increases with viscosity. Gold et al. provide further $\alpha$ values for a range of lubricant types [16]. From the EHD film thickness equations it might initially be inferred that the larger the $\alpha$-value the better (the thicker the EHD film). This does not turn out to be the case for two reasons. One is that there is a broad correlation between pressure-viscosity coefficient and viscosity index, with base fluids having high $\alpha$-value tending to have low VI and vice versa. The origin of this correlation is discussed in [17]. In practical terms it turns out to be more important to have a high VI (and thus maintain high dynamic viscosity at high temperature) rather than have high $\alpha$-value. Secondly, there is a tendency of fluids that high $\alpha$-value to give high EHD friction and vice versa [18]. 


\section{EHD FILM THICKNESS - COMPLICATIONS}

The above equations to predict EHD film thickness have a number of limitations, some of which are outlined here.

\subsection{Starvation}

The EHD film thickness equations (18) to (23) assume that the contact is well-supplied by lubricant so that the inlet is completely filled. If there is not enough lubricant reaching the inlet then fluid entrainment is inhibited and "starvation" occurs. In practice, EHD contacts require very little lubricant supply to fill the inlet, so starvation is quite rare with liquid-lubricated contacts except in rolling element bearings operating at high speeds and low temperatures. However it occurs commonly with grease lubricated systems. Starvation has been studied experimentally using optical interferometry [19] and numerically [20]. Both suggest that starvation starts to occur when the inlet meniscus (where the fluid layers adhering to the two surfaces merge) occurs less at than $\sim 3 a$ in front of the contact inlet edge, ( $a$ is the contact semi-width or radius), though this increases to about $5 a$ at very high entrainment speeds. (In practice starvation usually only becomes really significant when the inlet meniscus approaches $0.5 a$ ). Equations have been produced to predict the consequent reduction in film thickness but these require knowledge of either the position of the inlet meniscus or the thickness of oil film adhering to the two surfaces and are thus of limited practical value.

\subsection{Inlet Shear Heating}

At very high entrainment speeds, especially when some sliding is present, the lubricant in the contact inlet becomes heated, which reduces its viscosity and thus the quantity of lubricant entrained. Correction equations for EHL film thickness have been determined and are discussed in a recent paper by Hili et al. [21].

\subsection{Transient EHL}

In many practical non-conforming contacts, the contact conditions vary over time. For example, in gears the load and entrainment speed vary during tooth engagement, while in cams, load, entrainment speed and reduced radius all vary during a cycle. In principle we need to solve the transient EHD problem with the full Reynolds equation allowing for a finite value of $\mathrm{d} h / \mathrm{d} t$ [22]

$$
\frac{\partial}{\partial x}\left\{\frac{h^{3}}{\eta} \frac{\partial p}{\partial x}\right\}+\frac{\partial}{\partial y}\left\{\frac{h^{3}}{\eta} \frac{\partial p}{\partial y}\right\}=12\left\{U \frac{d h}{d x}+\frac{d h}{d t}\right\}
$$

Fortunately, however, the influence of this "squeeze term" on film thickness can usually be neglected and the steadystate equations (18)-(23) can be used in most cyclically-varying conditions using the instantaneous speed, load and geometry [23]. Exceptions are when there is a very abrupt increase of applied load or when reversal of entrainment occurs, as takes place in the contact between cams and tappets and in some constant velocity joints. When $U=0$, according to steady state EHD theory the minimum film thickness should fall to zero but in practice this does not occur since it takes a finite time for lubricant to be squeezed from the contact. Hooke has analysed the minimum EHD film thickness in a line contact during reversal [24]. He identifies two possible locations of the lowest film thickness. The first occurs just after entrainment reversal at what was the original inlet to the contact. The second is found some time later after the original exit restriction has moved through the conjunction. Hooke refers to these as "inlet" and "exit" minimum respectively and both must be calculated to determine which is smallest.

Hooke provides equations for both minima in terms of three non-dimensional groups as follows.

$$
\text { Inlet minimum; } \frac{h_{o}}{R_{x}^{\prime}}=2.66 G_{o} G_{1} G_{3}^{-0.5}
$$

Exit minimum; $\frac{h_{o}}{R_{x}^{\prime}}=1.97 G_{o} G_{1}^{0.856} G_{3}^{-0.14}$

where $G_{o}=\left(\frac{\eta_{o}^{2} \dot{U} R_{x}}{W_{L}^{2}}\right)^{2 / 3}, G_{1}=\left(\frac{\alpha^{3} W_{L}^{5}}{\eta_{o}^{2} \dot{U} R_{x}^{4}}\right)^{1 / 3}, G_{3}=\left(\frac{W_{L}^{7}}{E^{3} \eta_{o}^{4} \dot{U}^{2} R_{x}^{5}}\right)^{1 / 6}$ and $\dot{U}$ is the rate of change of entrainment velocity (i.e. the entrainment acceleration).

\subsection{Inlet Shear Thinning}

The pressure and shear rate within an EHD contact inlet are not severe enough to cause shear thinning of most lubricants, so EHD film thickness can generally be predicted accurately from low shear rate viscosity, $\eta_{o}$. An important exception is for polymer solutions, as commonly found with engine and gear lubricants, which shear thin at quite low strain rate. In this case, shear thinning in the inlet results in a thinner EHD film thickness than predicted from low shear 
rate viscosity of the formulated oil. Some inlet shear thinning can also occur with base fluids that are based on relatively high MWt polymers. If shear thinning does occur, when calculating the effective $\alpha$-value using equation 29 , it is important to use a value $\eta_{\text {otest }}$ measured at high shear rate, preferably at $10^{6}-10^{7} \mathrm{~s}^{-1}$.

Bair has recently proposed an EHD film thickness correction for such lubricants, based on knowledge of how their viscosity varies with shear rate, their flow curves [25]. However this requires knowledge of the flow curves at elevated pressure which are rarely available. Generally it is simpler to measure the film-forming properties of polymer solutions directly using optical interferometry

\section{EHD FRICTION}

EHD friction is becoming increasingly important in the quest to increase the efficiency of mechanical transmissions and thus reduce energy consumption. Traditionally the term EHD traction is often used in place of EHD friction. However the two terms are synonymous and in this paper EHD friction is used throughout.

\subsection{EHD Friction Calculation}

As described in Equ. 6, there are two sources of friction in an EHD contact, rolling friction and sliding friction. Rolling friction occurs primarily in the inlet region of the contact and is only a significant proportion of the total for contacts operating at very low $S R R$ and with high viscosity oils. Sliding friction is determined by integrating the resistance to Couette shear of the lubricant film as indicated in second term of Equ. 6 and originates primarily from the heavilyloaded central region of the contact. Its calculation is complicated by a number of factors. Firstly we need an expression for the rheological response of the lubricant film to strain rate, which, in the high pressure contact, is highly non-Newtonian. The choice of appropriate equation is controversial and its use requires accurate viscometry data for the lubricant at high pressures. Secondly, at high SRRs there is a temperature rise of the lubricant film in the contact, so that a solution incorporating thermal behaviour (and requiring lubricant thermal conductivity values at high pressure) is needed.

There is a third and more fundamental issue, in that when viscosity varies with strain rate, as in practice it does in all EHL contacts where sliding is present, Reynolds equation in the form shown in equation 1 is not, in principle, correct. Instead a complex form of Reynolds equation that allows viscosity to vary through the thickness of the film, known as the generalised Reynolds equation, should be used [26]. Fortunately, because of the decoupling of film thickness and friction mentioned in section 5 of this paper, solution of equation 1 to calculate film thickness (and provide the regression equations (18) to (23)), followed by equation 6 to calculate the friction, generally provides a reasonably accurate approach.

Currently there are two models for how lubricants shear thin at the very high shear stresses present in EHD contacts and there is considerable debate as to which is most appropriate.

Eyring equation (sometimes called the Ree-Eyring equation) [27]

$$
\tau=\tau_{e} \sinh ^{-1}\left(\frac{\eta \dot{\gamma}}{\tau_{e}}\right)
$$

Carreau-Yasuda equation [28]

$$
\tau=\eta \dot{\gamma} \frac{1}{\left\lfloor 1+\left(\frac{\eta \dot{\gamma}}{\tau_{s}}\right)^{a}\right\rceil^{(1-n) / a}}
$$

where $\tau$ is the shear stress, $\dot{\gamma}$ the strain rate and $\eta$ the low shear rate viscosity at the prevailing pressure. $\tau_{e}, \tau_{s}, n$ and $a$ are constants with $\tau_{e}, \tau_{s}$ corresponding to shear stresses at which shear thinning starts to become significant.

Both models include an additional, viscoelastic response term (not shown), which is only significant at very low SRRs, and both also assume that the shear stress levels out when it reaches a "critical shear stress", $\tau_{c}$, which increases linearly with pressure.

The Eyring model fits EHD friction data very well and has been the preferred choice of researchers of EHL since the early days of the subject [29]. The Carreau-Yasuda model was developed by rheologists studying the shear thinning properties of polymer melts and solutions and has been advocated by those using high shear rate viscometry to study 
lubricant shear thinning. In practice both models can be made to fit experimental data closely by choice of appropriate constants.

The term $\eta$ in equations 33 and 34 refers to the low shear rate viscosity of the lubricant at the prevailing, high contact pressure. At low pressures this can be estimated from the Barus equation, Equ. 26, but at pressures above about 100 $\mathrm{MPa}$ many lubricants have a $\log$ (viscosity) versus pressure curve whose slope initially decreases but then increases above about $1 \mathrm{GPa}$ [30]. Thus to predict shear stress at high pressure, viscosity-pressure data is needed over the whole pressure range up to the maximum contact pressure.

In practice, all of the above means that it is presently much simpler to measure lubricant EHD friction properties in a test machine and apply these directly or via a simple curve-fitting exercise to predict of the EHD friction in machine components. This is described in the next section.

\subsection{EHD Friction Measurement}

EHD friction is generally measured using two types of device, a disc machine or a ball on rotating flat machine. These are shown schematically in Fig. 6. In the ball on rotating flat, the ball shaft is tilted to intercept the top surface of the flat at its rotating centre so as to minimise spin in the contact.

The key to both of these types of device is that the two bodies are rotated one against the other at different and variable speeds, $u_{1}$ and $u_{2}$, so any combination of sliding and rolling speed can be achieved. This enables friction to be studied as a function of sliding speed (and thus strain rate) at a fixed entrainment speed and thus EHD film thickness, which would not be possible if one body were held stationary with $u_{2}=0$
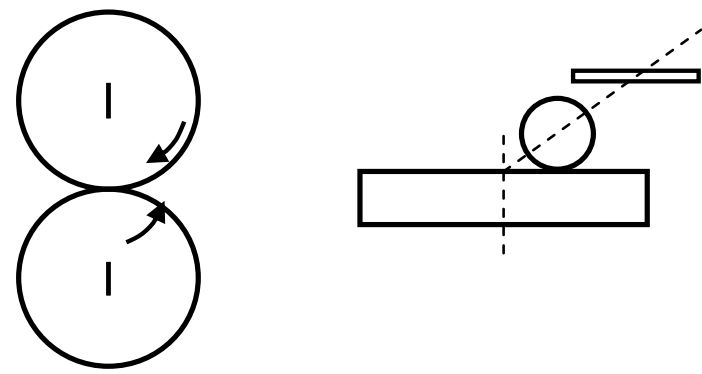

Fig. 6. Two common types of rolling-sliding contact for EHD friction measurement

Figure 7 shows a series of EHD friction curves (so-called "traction curves") in which entrainment speed, load and test temperature are all held constant while the slide-roll ratio is gradually increased from a very low to a high value. The entrainment speed was selected to be high enough to ensure there was a full EHD film $(2 \mathrm{~m} / \mathrm{s})$. These curves were obtained using a ball on flat minitraction machine (MTM) [31].

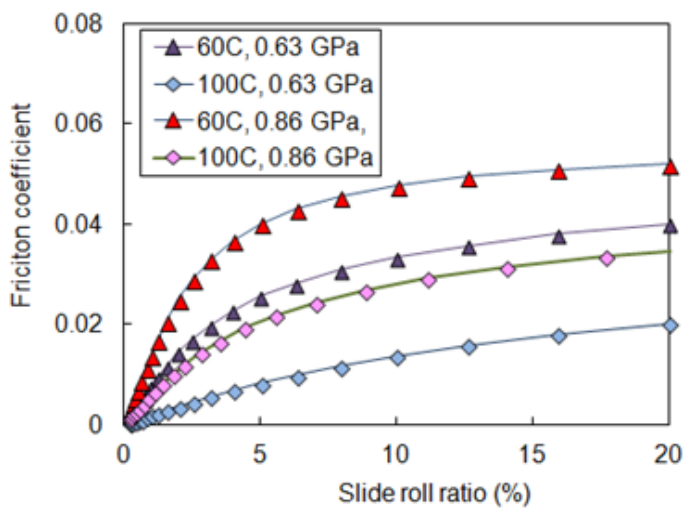

Fig. 7 Typical EHD friction coefficient versus $S R R$ curves, (lubricant is di-2-ethylhexylphthalate)

It can be seen that friction coefficient starts off at a very low value (close to zero in pure rolling) and increases rapidly, before levelling out at high slide-roll ratios. In general, EHD friction coefficient decreases with temperature and increases with pressure. These curves can be converted very easily into curves of mean shear stress versus strain rate and thus provide a description of the rheology of the EHD film. The mean shear stress, $\bar{\tau}$, is simply the friction force, $F$, divided by the area of contact, which for a circular, ball on flat contact is $\pi a^{2}$. Thus the mean shear stress is proportional to friction coefficient. The strain rate is the sliding speed divided by the EHD film thickness, $h_{c}$. So long as the entrainment speed is held constant, the sliding speed is proportional to the $\operatorname{SRR}\left(u_{s}=\operatorname{SRR} . U\right)$, while $h_{c}$ is constant $\left(h \propto U^{0.68}\right)$. Thus the strain rate, $u_{s} / h_{c}$, is proportional to the $S R R$. 
Figure 8 shows curves of mean shear stress versus strain rate calculated from the friction versus SRR curves in Fig. 7. The curve shapes look different from Fig. 7 simply because the strain rate axis has been plotted logarithmically to spread out the low SRR measurements. The shape of these curves, with an increasing gradient at low strain rates, followed by a linear region, closely resembles the $\sinh ^{-1}$ form of shear stress versus strain rate curve, predicted by the Eyring viscosity model and this is one reason it has become a favoured EHD rheological model of shear thinning.

Table 5 compares measured traction coefficients at 5\% SRR, two temperatures and two mean contact pressures (load over contact area) for the same series of base oils whose effective pressure viscosity coefficients were listed in Table 4.

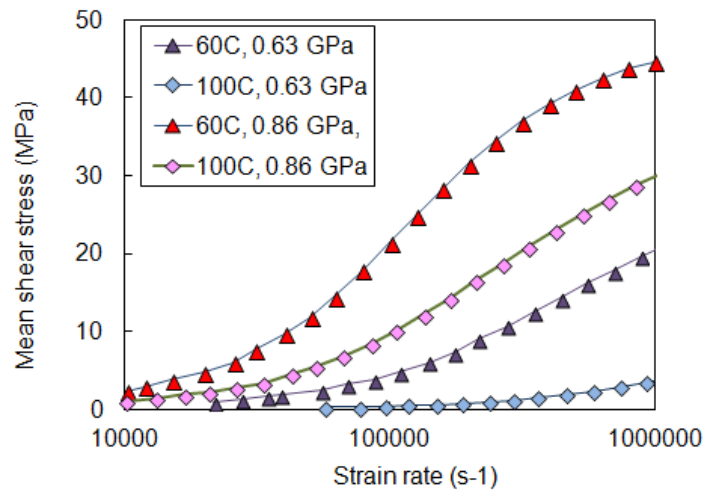

Fig. 8 Mean shear stress versus strain rate curves

\begin{tabular}{|l|c|c|c|c|}
\hline & $\begin{array}{c}60^{\circ} \mathrm{C}, \\
1.5 \mathrm{GPa}\end{array}$ & $\begin{array}{c}60^{\circ} \mathrm{C}, \\
2.0 \mathrm{GPa}\end{array}$ & $\begin{array}{c}100^{\circ} \mathrm{C}, \\
1.5 \mathrm{GPa}\end{array}$ & $\begin{array}{c}100^{\circ} \mathrm{C}, \\
2.0 \mathrm{GPa}\end{array}$ \\
\hline Di-ethylhexyl-phthalate & 0.063 & 0.072 & 0.049 & 0.060 \\
\hline Bis(2-ethyl hexyl)-sebacate & 0.044 & 0.057 & 0.025 & 0.040 \\
\hline Di-isodecyl azelate & 0.055 & 0.068 & 0.034 & 0.051 \\
\hline NPG di-oleate & 0.051 & 0.065 & 0.034 & 0.049 \\
\hline NPG di-isostearate & 0.040 & 0.055 & 0.024 & 0.039 \\
\hline PAO & 0.045 & 0.059 & 0.031 & 0.045 \\
\hline PAO & 0.047 & 0.060 & 0.032 & 0.047 \\
\hline PAO & 0.049 & 0.064 & 0.038 & 0.050 \\
\hline Group I MO & 0.062 & 0.072 & 0.047 & 0.060 \\
\hline Group II MO & 0.063 & 0.073 & 0.049 & 0.060 \\
\hline Group III MO & 0.055 & 0.066 & 0.036 & 0.051 \\
\hline Group III MO & 0.057 & 0.069 & 0.041 & 0.055 \\
\hline Castor Oil & 0.043 & 0.059 & 0.025 & 0.039 \\
\hline
\end{tabular}

Table 5. EHD friction coefficients at $0.05(5 \%) S R R$

These friction coefficients can be used as rough estimates of EHD traction for these lubricants, but do not allow friction coefficient to be dependent on slide-roll ratio. This can be achieved, however, by fitting an equation of the form;

$\bar{\tau}=\tau_{e} \sinh ^{-1}\left(\frac{\eta_{e} e^{\overline{\alpha p}} \dot{\gamma}}{\tau_{e}}\right)$

to experimental EHD friction curves, to determine the two constants $\tau_{e}$ and $\bar{\alpha}$, where the latter is an average pressureviscosity coefficient. Both constants depend on temperature and contact pressure, so EHD friction has to be measured, and $\tau_{e}$ and $\bar{\alpha}$ calculated at a range of temperatures and pressures.

Values of $\tau_{e}$ and $\bar{\alpha}$ determined from EHD friction measurements on di-ethylhexylphthalate, based on Equ. 32, are listed in Tables 6 and 7. Using these, with interpolation when necessary, EHD friction coefficients can then be determined from;

$\mu=\frac{\tau_{e}}{\bar{p}} \sinh ^{-1}\left(\frac{\eta_{o} e^{\overline{\alpha p}} U . S R R}{h_{c} \tau_{e}}\right)$ 


\begin{tabular}{|c|c|c|c|}
\hline & $60^{\circ} \mathrm{C}$ & $80^{\circ} \mathrm{C}$ & $100^{\circ} \mathrm{C}$ \\
\hline $0.63 \mathrm{GPa}$ & 10.5 & 8.3 & 6.6 \\
\hline $0.86 \mathrm{GPa}$ & 22.4 & 17.3 & 13.4 \\
\hline $1.5 \mathrm{GPa}$ & 25.8 & 20.7 & 17.9 \\
\hline $2.0 \mathrm{GPa}$ & 33.8 & 29.2 & 24.7 \\
\hline
\end{tabular}

Table 6. Values of $\tau_{\mathrm{e}}$ in MPa at various mean film temperatures and mean contact pressures

\begin{tabular}{|c|c|c|c|}
\hline & $60^{\circ} \mathrm{C}$ & $80^{\circ} \mathrm{C}$ & $100^{\circ} \mathrm{C}$ \\
\hline $0.63 \mathrm{GPa}$ & 13.1 & 12.0 & 10.9 \\
\hline $0.86 \mathrm{GPa}$ & 11.6 & 11.9 & 11.9 \\
\hline $1.5 \mathrm{GPa}$ & 7.9 & 7.9 & 7.8 \\
\hline $2.0 \mathrm{GPa}$ & 6.5 & 6.5 & 6.5 \\
\hline
\end{tabular}

Table 7. Values of $\bar{\alpha}$ in $\mathrm{GPa}^{-1}$ at various mean film temperatures and mean contact pressures

At high shear stresses and SRRs, the temperature of the EHD film in the contact starts to increase above the bulk temperature due to frictional heating and this results in the friction curves levelling out and then, in some cases, actually falling. The latter occurs particularly with high viscosity oils that form thick films, at very high pressures and for fluids with very high EHD friction coefficients. The total mean temperature the EHD film can be estimated as the sum of three components, the bulk temperature of the surfaces before they reach the contact, the mean temperature rise of the surfaces in the contact, $\Delta T_{\text {surf }}$, often called the flash temperature, and how much hotter the oil film becomes than the bounding surfaces, $\Delta T_{\text {oil }}$.

$T=T_{b u l k}+\Delta T_{\text {surf }}+\Delta T_{\text {oil }}$

For an EHD contact of two bodies of the same material, the first term can be approximated to;

$\Delta T_{\text {surf }}=\left(\frac{a}{\pi K_{\text {surf }} \rho c U}\right)^{0.5} \bar{\tau} u_{s}$

where $K_{\text {surf }}, \rho$ and $c$ are the thermal conductivity, density and specific heat of the solids, while the second is;

$\Delta T_{\text {oil }}=\frac{1}{8 K_{\text {oil }}} \bar{\tau} u_{s} h_{c}$

where $K_{\text {oil }}$ is the thermal conductivity of the lubricant (at the mean contact pressure) [32].

The product of the mean shear stress and the sliding speed, $\bar{\tau}_{s}$, is simply the heat generated per unit contact area. These equations can be used to correct EHD friction so long as the temperature dependence of $\tau_{e}$ and $\bar{\alpha}$ in Equ. 33 is known. The film thickness $h_{c}$ used in Equ. 36 must not however be corrected for temperature rise since it is determined in the inlet, where little if any heating normally occurs.

\section{EHL OF ROLLING BEARINGS}

EHL film thickness and friction calculations are important in rolling bearing design but are generally carried out by bearing suppliers and embedded in the bearing selection handbooks provided by these suppliers. Once a bearing size has been selected based on the bearing load and space constraints, bearing handbooks indicate the "rated viscosity". This is the viscosity required to generate a sufficiently large lambda ratio (ratio of EHD film thickness to surface roughness) and is extracted from handbook charts produced from EHD film calculations made by the bearing supplier, who assumes a representative $\alpha$-value.

Although bearing handbooks relieve the user from the need to calculate of EHD film thickness it is still sometimes instructive to make this calculation, so a simple example is provided here.

Consider a deep groove ball bearing having balls of radius $12.0 \mathrm{~mm}$ and an inner groove radius $18 \mathrm{~mm}$. The geometry (not to scale) is shown in Fig. 9. 


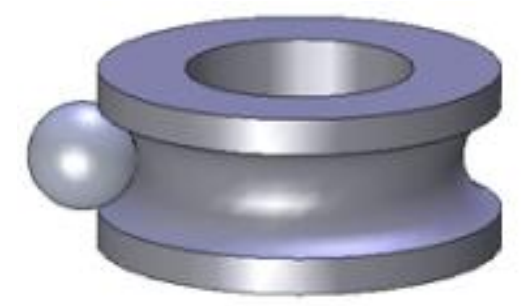

Fig. 9. Ball bearing-inner raceway geometry

The radius of the inner groove along the axial direction is $12.5 \mathrm{~mm}$ and is concave. The most heavily loaded ball (the one directly opposite the applied shaft load) has a load $W$ of $1.0 \mathrm{kN}$; (this can be evaluated from the applied bearing load using $W \approx \frac{5 W_{\text {bearing }}}{N}$ where $N$ is the number of balls in the bearing). What is the minimum EHD film thickness at the inner race if this race rotates at $1000 \mathrm{rpm}$ and the oil has viscosity of 0.01 Pas and pressure viscosity coefficient, $\alpha^{*}$ is $15 \mathrm{GPa}^{-1}$ ? The outer raceway is stationary.

$W, \eta_{\mathrm{o}}$ and $\alpha^{*}$ are known, so solution using Equ. 22 requires evaluation of $E^{\prime}, R_{x}, R_{y}$ and $U$. For $E=220 \mathrm{GPa}$ and $v=$ $0.3, E^{\prime}$ can be calculated from Equ. 8 to be $E^{\prime}=231 \mathrm{GPa} . R_{x}$ and $R_{y}$ can be calculated from Equ. 10 and 11 with $R_{x l}=$ $12 \mathrm{~mm}, R_{x 2}=18 \mathrm{~mm}, R_{y l}=12 \mathrm{~mm}$ and $R_{y 2}=-12.5 \mathrm{~mm}$ (negative since it is concave). This gives $R_{x}=7.2 \mathrm{~mm}$ and $R_{y}=$ $300 \mathrm{~mm}$.

For rolling bearings, calculation of the entrainment speed $U$ is often the most complicated stage since the ball moves around the bearing as it rotates, so the contacts between the ball and the raceways move in space. This is most easily dealt with by assuming that the ball rotates around the bearing with an (unknown) circumferential angular speed $\omega_{c}$. This rotational speed is then subtracted from the inner and outer raceway angular speeds as shown in Fig. 10, to effectively create a frame of reference in which the ball stationary.
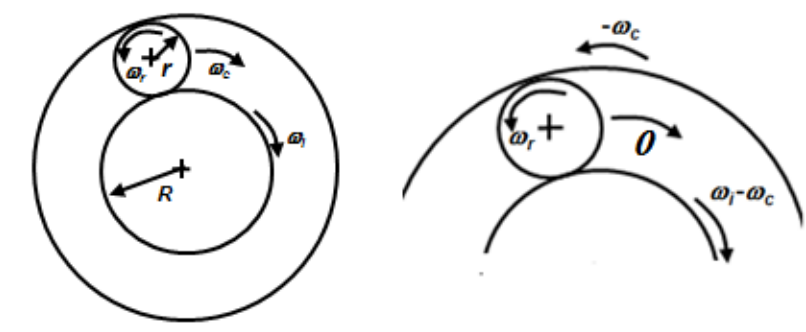

Fig. 10. Transformed rotational speeds of the elements in the rolling bearing

Now we assume that there is pure rolling at both the inner and outer raceway/ball contacts so;

At inner raceway contact; $r \omega_{r}=R\left(\omega_{i}-\omega_{c}\right)$

At outer raceway contact; $r \omega_{r}=(R+2 r) \omega_{c}$

Simultaneous solution of these two equations eliminates the unknown $\omega_{c}$ and gives;

$\omega_{r}=\frac{R}{2 r}\left(\frac{R+2 r}{R+r}\right) \omega_{i}$

so

$U=\frac{r \omega_{r}+r \omega_{r}}{2}=\frac{R \omega_{i}}{2}\left(\frac{R+2 r}{R+r}\right)$

For the bearing contact above this gives $U=1.35 \mathrm{~m} / \mathrm{s}$.

All of these values, $W, U, R_{x}, R_{y}, E^{\prime}, \eta_{\mathrm{o}}$ and $\alpha$ can now be inserted in equation 22 . This yields a minimum EHD film thickness of $h_{o}=65 \mathrm{~nm}$

A recent study measured the viscometric, film forming and boundary and EHD friction properties of a wide range of lubricants and correlated these with the temperature rise in an ARKL test [33]. This test measures how hot a loaded thrust bearing becomes after two hours operation; the smaller the temperature rise the lower the frictional and other losses. It was found that low ARKL temperature correlated far more closely with EHD friction measured at low SRR than with any other property of the lubricant, indicating the importance of EHD friction on rolling bearing efficiency. 
Bearing handbooks still tend to estimate bearing EHD friction in a quite rudimentary way though some have separate values for the rolling friction, sliding friction, drag and seal friction. In most rolling bearings, sliding friction originates from small amounts of micro-slip in the finite-sized EHD contact and thus represents EHD friction at a very low SRR (probably $<0.1 \% S R R$ ). For low viscosity lubricants, sliding friction tends to be larger than rolling friction but for very high viscosity lubricants the opposite is the case [34].

\section{EHL OF GEARS}

EHD lubrication analysis of gears is complicated by the relatively complex geometry and kinematics of gear pairs. To estimate EHD film thickness it is necessary to determine the radii of the surfaces, load, entrainment speed and sliding speed at the contact point of interest. These vary during tooth engagement and depend in a non-trivial way on the gear design. For this reason, most gear design and gear lubricant selection manuals tend to be based either on rule of thumb or, like bearing selection handbooks, bury any EHD calculations in selection charts and tables. Exceptions are the Mobil EHL Guidebook [35] and the AISI/AGMA gear rating publication [36]. The Mobil EHL Guidebok, first published in 1979, provides equations for calculating the pitch line EHD film thickness in a range of gear types in terms of gear geometry, operating conditions and a "Lubricant Parameter". The latter is simply the product of dynamic viscosity and effectve $\alpha$-value (determined from EHL film thickness measurements) and the Guidebook charts this property as a function of temperature for all the company's gear lubricants so that users can select a lubricant that provides an acceptable film thickness for a given gear set. A paper based on the approach used in the Guidebook can be found in [37].

This Guidebook uses an approximate form of the EHD film thickness equations that assumes that film thickness depends on viscosity and pressure viscosity coefficient to the same power, so that they can be lumped as one value (rather than separate powers as in Equs. (18) to (23)). But both it and [34] provide a useful compilation of gear contact conditions for film thickness determination in gears. For a parallel, external axis helical gear, for example, they show that

$$
\begin{aligned}
& U=\frac{2 \pi N_{G} R_{G}}{60} \sin \phi_{n} \cos \varphi \\
& W_{L}=\frac{T_{G}}{R_{G} F \cos \phi_{n} \cos ^{2} \varphi} \\
& R_{x 1}=\frac{R_{G} \sin \phi_{n}}{\cos ^{2} \varphi} \\
& R_{x 2}=\frac{R_{P} \sin \phi_{n}}{\cos ^{2} \varphi}
\end{aligned}
$$

where $R_{G}$ the pitch radius of the gear wheel, $R_{P}$ the pitch radius of the pinion, $N_{G}$ the gear wheel $\mathrm{rpm}, T_{G}$ the gear wheel torque, $F$ the face width, $\phi_{n}$ the normal pressure angle and $\varphi$ the helix angle. If $\varphi=0$ then the above equations are applicable to spur gears. (Note that the gear ratio $G=N_{P} / N_{G}=R_{G} / R_{P}$ and $C=R_{G}+R_{P}$.) The torque can be found from the power rating; $T_{G}=60 P / 2 \pi N_{G}$ where $P$ is the power in $\mathrm{W}$. It is straightforward to calculate EHD film thickness from the line contact equations (18) and (19) once these values of entrainment speed, load and radius are known. Clearly the equations (46) and (47) can also be used to determine contact pressure.

The above equations refer only to the pitch line location, where there is pure rolling and thus EHD friction is negligible. Away from this pitch line there is sliding between the two gears and to estimate EHD friction the sliding speed is required. Reasonably simple equations to determine contact conditions including sliding speed, in a form suitable for EHD analysis for gears, can be found in [36]. It should be noted that the above analysis assumes bulk isothermal conditions. In gear systems, heat generated due to churning and EHD friction often has a marked effect on inlet temperature and thus on inlet viscosity and film thickness., This can lead to film thickness being much less sensitive to entrainment speed and much more dependent on load than predicted by the EHD film thickness equations [38].

\section{CONCLUSIONS}

This paper has reviewed the basics of EHL with respect to the latter's practical application to engineering components. It has shown how film thickness and friction can be calculated based on reasonably simple equations as alternatives to full solution of the EHD problem. These equations require lubricant rheological parameter values that can, in principle, be determined by high pressure and high shear rate viscometry. However, in practice, it is currently much simpler to measure these parameters using EHD film thickness and EHD friction data from simple laboratory bench tests. From 
these equations and an understanding of EHD lubrication it is then reasonably straightforward to estimate EHD film thickness and friction in machine components such as gears and rolling bearings.

\section{REFERENCES}

1. Reynolds, Osborne. "On the theory of lubrication and Its application to Mr. Beauchamp Tower's experiments, including an experimental determination of the viscosity of olive oil." Proc. Roy. Soc. Lond. 1886; 40, pp. 191203.

2. Mohrenstein-Ertel, A. Die Berechnung der hydrodynamischen Schmierung gekrümmter Oberflächen unter hoher Belastung und Relativbewegung. PhD Thesis first published in 1945 in Russian under the name A.M. Ertel. Fortschr.-Ber. VDI-Z. Reihe 1, Nr 115, publ. 1984, VDI Verlag, Düsseldorf. Translated as Grubin, A.N., Vinogradava, I.E. Central Scientific Research Institute for Technology and Mechanical Engineering, Book No. 30, Moscow, 1949. D.S.I.R Trans. No. 337.

3. Spikes, H.A., “Sixty years of EHL”. Lubr. Sci. 2006;18, pp. 265-291.

4. Hamrock, B.J., Schmid, S.R. Jacobson, B.O. "Fundamentals of Fluid Film Lubrication $2^{\text {nd }}$ Edition. Publ. Marcel Dekker 2004.

5. Johnson, K.L., Contact Mechanics, Cambridge University Press (1985).

6. Dowson, D., Higginson, G.R. "A numerical solution to the elastohydrodynamic problem." J. Mech. Eng. Sci. 1959; 1, pp. 6-15.

7. Dowson, D., Toyoda, A., "A central film thickness formula in EHD line contacts." Proc 5th Leeds-Lyon Symp. Elastohydrodynamic Lubrication and Related Topics, pp. 60-65, 1978, publ. MEP Press, London, 1979.

8. Chittenden, R.J., Dowson, D., Dunn, J.F. and Taylor, C.M., "A theoretical analysis of the isothermal elastohydrodynamic lubrication of concentrated contacts. I. Direction of lubricant entrainment coincident with the major axis of the Hertzian contact ellipse.” Proc. Roy. Soc. Lond. Series A 1985; 397, pp. 245-269.

9. Chittenden, R.J., Dowson, D., Dunn, J.F. and Taylor, C.M., "A theoretical analysis of the isothermal elastohydrodynamic lubrication of concentrated contacts. II General case, with lubricant entrainment along either principal axis of the Hertzian contact ellipse or at some intermediate angle.” Proc. Roy. Soc Lond. Series A 1985; 397, pp. 271-294.

10. Nijenbanning, G., Venner, C.H., Moes,H., "Film thickness in elastohydrodynamically lubricated elliptic contacts", Wear 1994; 176, pp. 217-229.

11. Johnson, K.L., "Regimes of elastohydrodynamic lubrication.” J. of Mech. Eng. Sci.1970; 12, pp. 9-16.

12. Esfahanian, M., Hamrock, B.J., "Fluid-film lubrication regimes revisited.” Trib. Trans. 1991; 34, pp. 628-632.

13. Spikes, H.A. "Thin films in elastohydrodynamic lubrication: the contribution of experiment." Proc. I.Mech.E. Series J 1999; 213, pp. 335-352.

14. Zhang, J., Drinkwater, B.W., Dwyer-Joyce, R.S., “Acoustic measurement of lubricant-film thickness distribution in ball bearings.” J. Acoust. Soc. Am. 2006; 119, pp. 863-871.

15. Roelands, C.H.A. "Correlational aspects of the viscosity-temperature-pressure relationships of lubricating oils. Chapter 13" PhD thesis, University of Delft, 1966 (http://repository.tudelft.nl/).

16. Gold, P.W., Schmidt, A., Dicke, H., Loos, J., Assmann, C., "Viscosity-pressure-temperature behaviour of mineral and synthetic oils." J. Synth. Lubr. 2001; 18, pp. 51-79.

17. Spikes, H.A. “Thermodynamic approach to viscosity.” Trib. Trans. 1990; 33, pp. 140-148.

18. Smeeth, M., Gunsel, S., Korcek, S.G. and Spikes, H.A. "The elastohydrodynamic friction and film-forming properties of lubricant base oils." Trib. Trans. 1999; 42, pp. 559-569.

19. Wedeven, L.D., Evans, D., Cameron, A., “Optical analysis of ball bearing starvation.” Trans. ASME J. Lubr. Techn. 1971; 93, pp. 359-361. 
20. Hamrock, B.J., Dowson, D., "Isothermal elastohydrodynamic lubrication of point contacts. Part IV—starvation results.” Trans ASME J. Lubr. Techn. 1977; 99, pp.15-23.

21. Hili, J., Olver.A.V., Edwards, S., Jacobs, L., "Experimental investigation of elasto-hydrodynamic (EHD) film thickness behavior at high speeds.” Trib. Trans. 2010; 53, pp. 658-666.

22. Hamrock B.J., Dowson, D. Ball Bearing Lubrication. The Elastohydrodynamics of Elliptical Contacts. John Wiley \& Sons (1981).

23. Hooke, C.J., "The minimum film thickness in lubricated line contacts during a reversal of entrainment - general solution and the development of a design chart.” Proc I.Mech.E. Series J 1994; 208, pp. 53-64.

24. Hooke, C.J., Dynamic effects in EHL contacts. Proc. of the 29th Leeds-Lyon Symposium on Tribology, Tribological Research and Design for Engineering Systems, Tribology Series Vol. 41, pp. 69-78, (2003), Elsevier Press.

25. Habchi, W., Bair, S., Qureshi, F., Covitch, M., “A film thickness correction formula for double-Newtonian shearthinning in rolling EHL circular contacts.” Trib. Lett. 2013; 50, pp. 59-66.

26. Dowson, D., “A generalized Reynolds equation for fluid-film lubrication.” Intern. J. of Mech. Sci. 1962; 4, pp. 159-170.

27. Evans, C.R., Johnson, K.L., “The rheological properties of elastohydrodynamic lubricants.” Proc. I.Mech.E. Series C 1986; 200, pp. 303-312.

28. Bair, S., "Measurement of real non-Newtonian response for liquid lubricants under moderate pressures." Proc. I.Mech.E. Series J 2001; 215, pp. 223-233.

29. Hirst, W., Moore, A.J. "Elastohydrodynamic lubrication at high pressures. II - Non-Newtonian behaviour." Proc. Roy. Soc. Lond. Series A 1979; 365, pp. 537-565.

30. Bair, S., Kottke, P. "Pressure-viscosity relationships for elastohydrodynamics." Trib. Trans. 2003; 46. pp. 289295.

31. PCS Instruments. (http://pcs-instruments.com).

32. Archard J.F., The temperature of rubbing surfaces." Wear 1959; 2, pp. 438-455.

33. Yang, S., Reddyhoff, T., Spikes.H., "Influence of lubricant properties on ARKL temperature rise and transmission efficiency.” Trib. Trans. 2013; 56, pp. 1119-1136.

34. Ioannides E., Morales, G.E., Handbook of Lubrication and Tribology Vol. II Theory and Design, $2^{\text {nd }}$ Edition Chapter 49, Lubrication of Rolling Element Bearings, ed. Robert W Bruce, publ. CRC Press 2012.

35. Mobil, Mobil EHL Guidebook, publ. Mobil Oil Corp. NY 1979.

36. AGMA, American National Standard, Fundamental Rating Factors and Calculation Methods for Involute Spur and Helical Gear Teeth. ANSI/AGMA 2101-C95, publ. AGMA1995.

37. Jackson, A., Rowe, C.N., “Application of EHL theory to gear lubrication.” SAE Tech. Paper 800670, (1980).

38. Olver, A.V. "Gear lubrication - a review." Proc. I.Mech.E., Series J 2002; 216, pp. 255-267. 